\title{
Nephro Update Europe 2017
}

\author{
Nina Kesel \\ med update europe $\mathrm{GmbH}$, Wiesbaden, Germany
}

\section{Keywords}

Nephro Update Europe · Chronic kidney disease · Iron management · Obesity · Dialysis · Risk factor · Hypertension · Acute kidney injury · Urgent-start peritoneal dialysis .

Peritoneal dialysis

\section{About Nephro Update Europe}

A first-of-its-kind CME event premiered in Vienna at the beginning of October, updating delegates on recent developments across the broad spectrum of nephrology.

European leaders whose expertise lie in areas, such as diabetic nephrology, cardiovascular disease, glomerulonephritis, genetic disease, and much more, spent 2 compact days summarizing international literature and presenting the most recent cutting-edge developments. The scientific program and speakers were determined by cochairs Kai-Uwe Eckardt from Charité Berlin and Ton Rabelink from Leiden University Medical Center in the Netherlands.

In particular, trial and study results published in peerreviewed journals during the past year were discussed and practical, relevant take-home messages were presented with the goal of impacting daily patient care in a positive way.

Generous amounts of time were given to informal, interactive discussions and $\mathrm{Q}+\mathrm{A}$ during the lectures directly with the speakers. A Digital Lounge displayed ePoster

\section{KARGER}

(C) 2017 S. Karger AG, Basel submissions, and 3 winners were chosen by the event cochairs for having presented outstanding work results. Over 300 delegates from 40 countries attended the premiere at the Academy of Sciences in the heart of Vienna, with many already looking forward to next year's edition in Budapest.

Below is a glimpse into some of the information presented, on the topics of Chronic Kidney Disease (KaiUwe Eckardt), Renal Anemia (Iain Macdougall), Hypertension (A.J. Rabelink), Acute Kidney Injury (Patrick Murray), and Peritoneal Dialysis (An De Vriese). All presentations are available online for participants, for up to 1 year after the event.

\section{Risk Factors of Chronic Kidney Disease - Kai-Uwe Eckardt (Germany)}

More than $10 \%$ of the human population suffers from chronic kidney disease (CKD). Kidney failure and cardiovascular complications, even death, are very much connected, with many patients dying from cardiovascular disease (CVD) before kidney failure. The state-of-the-art definition of CKD acknowledges the competing risks between CVD and kidney failure, due to the disease's pro-

Meeting report of Nephro Update Europe 2017, October 6-7, 2017, Vienna, Austria.

Nina Kesel

med update europe $\mathrm{GmbH}$

Hagenauer Strasse 53

DE-65203 Wiesbaden (Germany)

E-Mailn.kesel@medupdate-europe.com 


\section{Obesity and CKD State of the Art}

- Prevalence of obesity increases worldwide

- Factors associated with obesity are associated with CKD

- Is obesity an independent risk factor for CKD?

Fig. 1. State of the art: obesity and CKD.

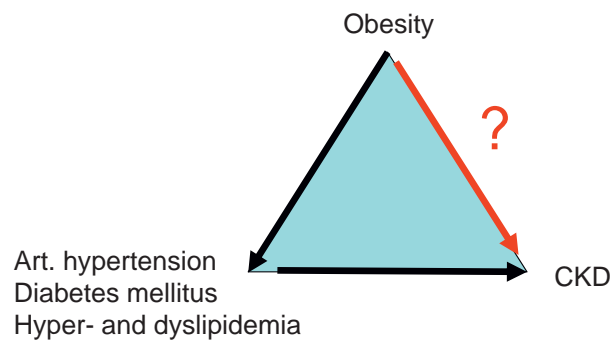

gressive loss of kidney function together with the development of CVD. The KDIGO (Kidney Disease: Improving Global Outcomes) CKD guideline from 2013, with its definition of CKD and the proposed staging according to cause, GFR (glomerular filtration rate), and albuminuria categories (CGA) is still valid.

Several studies over the past year determined that the overall trend of increasing CKD prevalence has probably reached a plateau. Various factors, including improved risk factor control and early detection, have probably all contributed to CKD no longer being on the rise.

Kai-Uwe Eckardt presented various recent studies focusing on the risk factors for the development and progression of $\mathrm{CKD}$, specifically the long-term effects of proton pump inhibitor (PPI) use, and the effects of obesity, diet, and sodium. He considered the data of an association between PPI use and CKD prevalence as a "smoking cannon." Results from the ARIC (Atherosclerosis Risk in Communities) study showed the effects of long-term PPI use, which were validated by the Geisinger Health System Replication Cohort [1]. ARIC is a large observational study in the United States, with over 10,000 participants and a median follow-up of 14 years. The results were as follows: patients with prolonged PPI use have a 30\% increased risk of CKD incidence and a 1.6-fold increased risk of acute kidney injury (AKI). Lazarus et al. [1] concluded that further research should be conducted to evaluate whether decreased PPI use decreases the risk of CKD.
In another study published this year, Xie and his team looked at almost 150,000 patients undergoing acid suppression therapy, using the Department of Veteran Affairs national databases, with a follow-up of 5 years and censored at the time of AKI development. The results showed that the long-term PPI users had a higher risk of an estimated glomerular filtration rate (eGFR) under 60 $\mathrm{mL} / \mathrm{min} / 1.73 \mathrm{~m}^{2}$ as compared to users of $\mathrm{H} 2$ blockers [2].

An interesting study out of Korea tackled the question of whether obesity is a direct risk factor for CKD or whether common risk factors exist that result in both CKD and obesity (Fig. 1). Jung et al. [3] studied a cohort of 41,000 people without CKD, looking not only at BMI but also metabolic health. Patients were categorized into 4 groups: metabolically healthy non-obese (MHNO), metabolically unhealthy non-obese (MUNO), metabolically healthy obese (MHO), and metabolically unhealthy obese (MUO). During the follow-up period of just over 3 years, 356 subjects developed incident CKD. The outcomes were that the MHO group, along with the MUNO group, were at increased risk of developing incident CKD, thereby concluding that metabolic health protects against CKD in obesity only partially, and both factors need to be considered when considering the risk of CKD [3].

In 2016, two studies looked at the effects of the DASH diet (Dietary Approaches to Stop Hypertension) and the impact of sodium and potassium as risk factors for CKD. In the ARIC study published in the American Journal of 
Fig. 2. Take-home message: risk factors for development and progression of CKD K.-U. Eckardt.

\section{Take-Home Messages Risk Factors for Development and Progression of CKD}

- Medication: avoid unnecessary long-term use of PPI

- Diet:

- Too much is not good; a "healthy" metabolic phenotype protects only partially in obesity

- DASH seems to protect

- Sodium is not as important as frequently presumed

- Diet soda is perhaps not as healthy as presumed
Kidney Disease in 2016 [4], the results were that with an increased use of a DASH-like diet (consisting of fruits and vegetables, less red meat, less sodium, and increased potassium) the risk of incident CKD decreased. These observational data are not surprising, but at the very least, they are reassuring.

Somewhat more intriguing data came from the PREVEND Study out of the Netherlands [5] looking at 5,000 people with normal kidney function over a follow-up period of 10 years. The study concluded that from the 872 patients who developed CKD, it was a low-potassium urinary output and not a high-sodium urinary output which was the risk factor in developing CKD (Fig. 2).

\section{Iron Management (Oral vs. i.v.) in Non-Dialysis Chronic Kidney Disease - lain Macdougall (UK)}

In a recently reported secondary analysis of the FINDCKD study, a detailed examination revealed no obvious safety signals with regard to iron management between the use of i.v. iron and oral iron. This 1-year, open label, multicenter, randomized trial of 616 patients with nondialysis CKD [6] found no difference in the frequency of infection-related adverse events (urinary tract infections, nasopharyngitis, influenza) between high-ferritin ferric carboxymaltose (FCM) (33.1\% rate of infection), low-ferritin FCM (34\%), and oral iron (30\%). When looking at

Nephro Update Europe 2017 serious adverse events, such as pneumonia, the results showed 3.9\% with high-ferritin FCM, 3.3\% with low-ferritin FCM, and 3.8\% with oral iron. Again, no obvious significant safety signals were seen.

Another secondary analysis by Iain Macdougall et al. [7] from the same study, published recently in $\mathrm{BMC} \mathrm{Ne}$ phrology, showed no deterioration in renal function across the high-ferritin FCM, low-ferritin FCM, and oral iron arms and no significant change in eGFR over the 12 -month study period in any treatment group. The analysis of 4 quartiles of i.v. iron dosing over 12 months revealed no differences or changes in renal function and no relationship between i.v. iron dose and any change in renal function. A limitation of this trial was that it used estimated, rather than measured, GFR.

The REVOKE study, published in 2015 [8], examined measured GFR; again a randomized trial with respect to i.v. iron versus oral iron. A large number of patients were screened for this trial, but from an initial 5,055 patients, only 69 were randomized to oral iron and only 67 to i.v. iron. Over the subsequent 24 months, a few patients were lost from the trial, with only 99 completing the study (50 to oral iron, 49 to i.v. iron). The trial was stopped early due to safety concerns, but Iain Macdougall believes that there were serious limitations to this study: the measured GFR showed identical rates of decline over the 2 years between i.v. and oral iron use. However, there was a greater incidence rate ratio (IRR) of serious adverse events 
(SAEs) in the group of patients receiving i.v. iron, and this is why the study was stopped prematurely.

During the congress, Iain Macdougall highlighted the highly significant difference between the unadjusted and adjusted IRR. The unadjusted IRR was unusual in that there was no statistical significance $(p=0.106)$, but when adjusted for baseline imbalance it became highly significant $(p<0.0001)$. The infection-related SAEs turned out exactly the same way; the unadjusted IRR between i.v. iron and oral use was $p=0.17$, but when adjusted, the IRR became $p<0.006$. This led to the concerns related to safety.

Regarding cardiovascular-related SAEs, the outcomes were similar; the unadjusted IRR was $p=0.033$ and when adjusted became $p<0.001$. The intervention period was 8 weeks after randomization with follow-up at 24 months. Many of the adverse events occurred at least 18 months after randomization, long after the intervention was complete. We question whether the adjustments provide the correct conclusions and whether the repeated events happening in the same patients (for oral iron: 19 patients, 36 events; for i.v. iron: 17 patients, 55 events), thereby driving the safety signal up, present the true picture of iron management.

Both the FIND-CKD and the REVOKE trials have informed the evidence base of oral iron versus i.v. iron in non-dialysis CKD. The safety concerns presented, Iain Macdougall believes, are due to study limitations. $\mathrm{He}$ concludes that the data from both studies mean that we can be fairly confident that i.v. iron is not toxic to the kidneys, despite earlier cellular work suggesting the contrary. The main message over the past few years is that i.v. iron is neutral in terms of renal function.

\section{Hypertension - A.J. (Ton) Rabelink (The Netherlands)}

A new, important development in hypertension therapy revolves around the blood pressure target. Over the past 2 years, a number of studies have been focused on redefining the blood pressure goals, and studying their impact, with surprising results.

Ton Rabelink acknowledged 2 large studies (Hope 3, SPRINT) that considered new blood pressure targets, lower than were anticipated, and lower than what the standard goals have been over the past years. The bottom line as shown by the outcomes is that we have to try harder to push to lower blood pressure levels. The data were very good, showing encouraging results regarding quality of life and health technology assessment aspects.
The SPRINT study identifies the new blood pressure target as $120 \mathrm{~mm} \mathrm{Hg} \mathrm{-} \mathrm{"a} \mathrm{phenomenal} \mathrm{task"} \mathrm{says} \mathrm{Rabelink.}$ A cohort of 9,361 patients with high risk for cardiovascular events, but without diabetes, had a mean age of 68 years. Blood pressure was pushed to $120 \mathrm{~mm} \mathrm{Hg}$ (in similar fashion to the ACCORD study from 2010) and doctors could decide themselves which drugs to use to achieve this goal. The surprising outcome is that it appears that this regime of intensive treatment resulted in a decrease in cardiovascular events and even in total mortality, which is something that is not often seen in trials [9].

What is also interesting is that one could surmise that a group of elderly patients undergoing a rigorous and intense drug program such as this one, which aims to lower blood pressure, would increase the incidence of falls and other injuries. A detailed analysis revealed that this was actually not the case, even in patients older than 75 years. In back-to-back papers published this year, Bress et al. [10] and Berlowitz et al. [11] looked at comparing costeffectiveness and willingness to pay, along with the quality of life for patients on intensive blood pressure-lowering therapy, respectively. The average age of the patients was 75 years of age in the patient satisfaction study, with data taken from the Veterans RAND 12-Item Health Survey [11]. Even with some side effects, such as increased dizziness, there was virtually no difference in quality of life scores for those in standard treatment versus patients in intensive treatment over a period of 48 months.

Taking into account a maximum amount of money acceptable for patients to pay for drug therapy, the cost-effectiveness study showed that when comparing lifetime best case (lifetime adherence and positive effects), best case ( 5 years adherence and positive effects for 15 years), base case (reduced adherence after 5 years and no effects at 15 years), and worst case (non-adherence and no effects at 5 years), there was very little difference with regards to the willingness-to-pay threshold [10].

A number of other studies over the past year resulted in similar conclusions regarding intensive blood pressure therapy in treating hypertension in the elderly. Overall, a target of reducing blood pressure to $120 \mathrm{~mm} \mathrm{Hg}$ reduces cardiovascular events and all-cause mortality. This intense treatment is cost-effective, does not reduce quality of life, and it is safe (Fig. 3).

Translating this into daily clinical practice, there are a few things that Ton Rabelink believes need to be further discussed. One important aspect is the method of blood pressure measurement, which was looked at by Agarwal [12]. In the SPRINT study, patients were seated for $5 \mathrm{~min}$ in a quiet room and then 3 oscillometric measurements 
Fig. 3. Take-home message: hyperten-

\section{Take-Home Message}

- Targeting a systolic blood pressure $<120 \mathrm{~mm} \mathrm{Hg}$ reduces $\mathrm{CV}$ death and all-cause mortality

- Is cost-effective and does not reduce QoL

- Is safe in (frail) elderly and mild CKD

The mean diastolic blood pressure achieved in the intensive group was $62 \mathrm{~mm} \mathrm{Hg}$, or $5 \mathrm{~mm} \mathrm{Hg}$ less than in the standard group.

were taken. The question of the study, and Rabelink's, is what sort of difference, if any, is there in this researchgrade data as compared to routine practice of measuring blood pressure.

Agarwal discovered a significant difference in result among 2 groups, those whose blood pressure was measured as in SPRINT, and those who were measured without being at seated rest. The research-grade systolic blood pressure was lower compared to the routine measurement and also lower than the daytime ambulatory systolic blood pressure, as recorded on the same day.

The conclusion is that the research-grade blood pressure target of $120 / 80 \mathrm{~mm} \mathrm{Hg}$ as in SPRINT is equivalent to a $132 / 92 \mathrm{~mm} \mathrm{Hg}$ measurement in a routine setting, and that in order to implement the SPRINT results in the wider population, the method of measurement would need to be the same as in the trial.

One last important note on this topic, which the SPRINT group just presented at ESC 2017 in September (still unpublished) shows that when the initial blood pressure is driven too low (blood pressure $>160 \mathrm{~mm} \mathrm{Hg}$ ), there is no longer a cardiovascular protective effect, and there is a small increase in all-cause mortality.

Another interesting development over the past year is that there appear to be good alternatives on the horizon to treat therapy-resistant hypertension. Previously, there had been renal denervation studies (Phase 3, Simplicity studies) that did not work, but they have now been rede-

Nephro Update Europe 2017 signed to focus on catheter technology, and repositioning, instead of drug therapy.

In very recent data from this past month, outcomes have determined that patients can have renal denervation without drug therapy [13]. Since medication adherence is a main problem in hypertension, this is quite an exciting finding and fits together with data emerging regarding barrier receptor activation. In a trial of 143 patients with a 5 -year follow-up, in approximately $25 \%$ it was possible to reduce the number of medications from a median of 6 to a median of 3 and there was a substantial reduction of blood pressure [14]. This therapy is very invasive, but "interesting conceptually" says Rabelink. The data look very promising and further studies, such as the Barostim Neo next-generation device study, are ongoing.

\section{Acute Kidney Injury Prevention - Patrick Murray (Ireland)}

There is a complex mixture of pre- and post-exposure risk with regard to AKI prevention.

Patrick Murray presented recent trials using a biomarker to define the point at which the risk of AKI is substantially increased, triggering a randomized prevention trial done with biomarker guidance.

The Zarbock group looked at high-risk cardiopulmonary bypass patients, randomized to standard care man- 
Fig. 4. Biomarker-guided prevention of cardiac surgery-associated AKI - Meersch et al. [15].

\section{Biomarker-Guided Prevention of Cardiac Surgery-Associated AKI}

\author{
Single-centre, RCT in high-risk CPB patients \\ [TIMP2].[IGFBP7] $\geq 0.3 @ 4$ h post-CPB \\ Randomized: standard care vs. KDIGO CT Surgery \\ Bundle \\ Primary endpoint: $\mathrm{AKI} \leq 72 \mathrm{~h}$ postop: \\ Control (99/138, 71.7\%) vs. intervention (76/138, \\ $55.1 \%) ; p=0.004$ \\ Stage 2/3 AKI: Control (44.9\%) vs. intervention \\ $(29.7 \%) ; p=0.009$ \\ No difference in RRT, MAKE 30,60, 90
}

agement versus a KDIGO cardiac surgery bundle (which includes avoiding contrast, nephrotoxins, and hyperglycemia and optimizing volume status and hemodynamic management, per protocol). The results showed that, using an AKI endpoint defined by the KDIGO criteria, there was a significant decrease in the incidence of AKI, along with a significant decrease of the more severe AKI stages.

In this single-center study, there was no difference in the incident renal replacement therapy, which is of comparatively low incidence in cardiac surgery, and no increase in major adverse kidney events (MAKE) at 30, 60, and 90 days (death, dialysis, or prolonged renal dysfunction) (Fig. 4). This positive trial determined enrollment by use of a kidney damage biomarker before randomization, and then linked it to a protocol to try and change the outcome. As more such biomarker-enriched trials, especially multicenter trials, are undertaken, the outcomes will be exciting to watch [15].

Another single-center study, also by Zarbock and his team, got very positive results using remote ischemic preconditioning (RIPC) preceding cardiac surgery to try and decrease the incidence of AKI. The short-term outcomes showed a significant decrease in AKI incidence overall, through all stages, along with less renal replacement therapy, and in a more recent follow-up study of this cohort, medium-term outcomes were also positive at 90 days [16, 17].
However, in 2 other recent larger, multicenter trials of RIPC in cardiac surgery, the outcomes were negative or showed no impact; a disappointing series of results.

The SPLIT trial (Saline vs. Plasma-Lyte for ICU Fluid Therapy) from 2015 is the only such trial which has been completed. The large, randomized trial compared saline to plasma-lyte and had a very complex, double-crossover design. In the end, the results were negative; there was no evidence from this trial that either AKI or mortality improved with the use of saline or plasma-lyte [18]. There continue to be much bigger, more complex trials with these agents going on now and so the jury is still out. To this date, there is still no proven way to decrease the incidence of AKI or improve other clinical outcomes with specific fluid choices, apart from avoidance of certain fluids in patient subsets (hetastarch in sepsis, albumin in traumatic brain injury), as reviewed.

Early goal-directed therapy (EGDT) was made a requirement in the United States to treat patients with sepsis several years ago, largely based on a single-center trial (the Rivers protocol), because it was shown to improve mortality in a single center. What has since followed is that British, Australian, New Zealand, and European trials have tried to reproduce the results on a multicenter basis.

Taken together, when looking at mortality or renal replacement therapy (incidence and duration or any other meaningful measure), there was no benefit to EGDT. 
Fig. 5. Large-cohort urgent-start PD - Xu et al. [22]. Note: dialysate volume of most patients $(80.5 \%)$ at 1 month was $<2 \mathrm{~L}$.

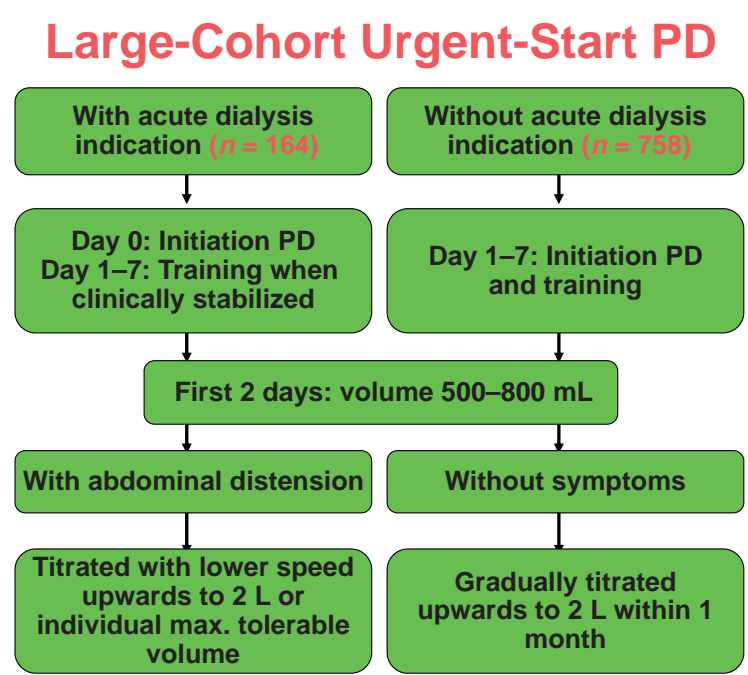

Nephro Update Europe 2017
EGDT requires replacement of a central line, use of aggressive transfusion, and aggressive use of vasoactive drugs, and in some of these trials there was evidence of harm. The 3 critical care networks agreed on the same endpoints, pooling their data in a patient-level meta-analysis which was published this year [19]. From these patient-level data, it is easy to conclude that there simply is not a signal, in terms of mortality or renoprotection, to show a benefit for using EGDT. Appropriate early volume resuscitation and rapid administration of antibiotics and sepsis source control remain the cornerstones of sepsis management, including AKI prevention and therapy, in this devastating syndrome. The disappointing impact of EGDT on renal outcomes further suggests that the pathogenesis of septic AKI may be mediated by tissue injury by circulating mediators, rather than primarily caused by hypoperfusion in severe sepsis.

\section{Urgent Start in Peritoneal Dialysis - An De Vriese (Belgium)}

The guidelines regarding peritoneal access state that peritoneal dialysis (PD) should be started at least 2 weeks after catheter insertion and that small volumes in the supine position can be infused if dialysis needs to begin earlier. Standard practice is that patients are often treated with hemodialysis to bridge the first 2 weeks if dialysis is required immediately [20].

An De Vriese presented a recent trial from Australia, one of the first randomized control studies to examine the ideal time to start PD. It compared the complication rate of PD initiation after catheter insertion at 1, 2, and 4 weeks. They found an increase in leaks in the patients that initiated PD 1 or 2 weeks after catheter insertion. However, overall technique survival was best in the 1 - and 2 -week groups. The conclusion is that if you have the time to wait, the optimal time to start PD is 2 weeks after PD catheter implantation [21].

In the cases where dialysis needs to be started earlier, we know from the literature that this is associated with a number of mechanical complications, such as pericatheter leak or catheter migration.

In the past year, a very large study with 900 patients presented an urgent-start protocol for manual PD, in contrast to most previous studies that adopted automated PD in the supine position (Fig. 5). All patients began with an equal volume of $500-800 \mathrm{~mL}$ over the first 2 days, which was then gradually titrated upwards to $2 \mathrm{~L}$ over approximately 1 month, provided the patients did not develop abdominal distension. In those cases, the infusion volume was kept at a tolerable level until the abdominal cavity adapted to the increased pressure, after which the volume was titrated upwards at a much lower speed, to $2 \mathrm{~L}$ or to 
Fig. 6. Abdominal wall complications - Xu

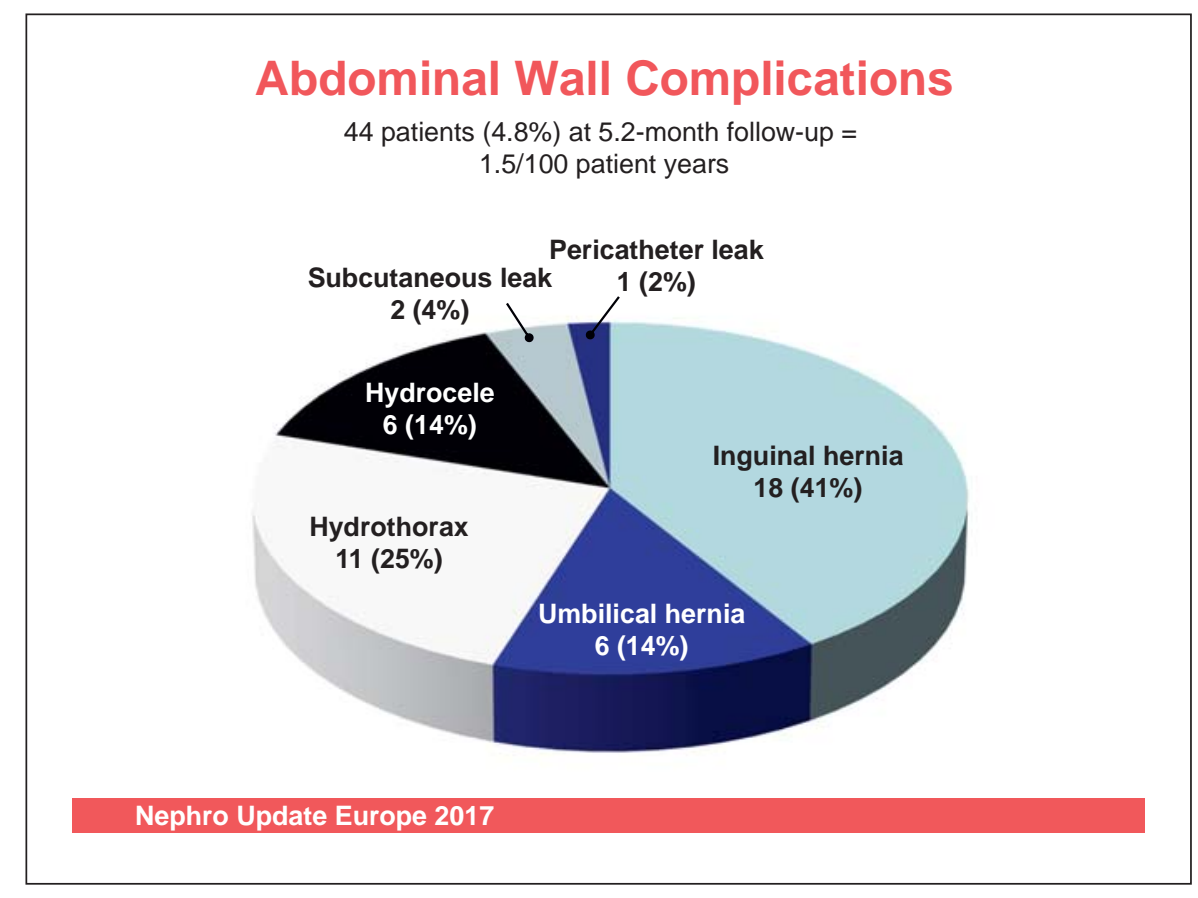

the maximum volume which the patient was able to tolerate. The break-in time was always shorter than 7 days and about $50 \%$ of the patients initiated PD within 2 days.

Only $5 \%$ of patients at the 5-month follow-up developed abdominal wall complications, with only 1 patient developing a pericatheter leak. The most common complication was hernia (Fig. 6). About $10 \%$ of patients had catheter complications, including obstruction, shift, and omental wrap, all of which occurred early on.

The main takeaway from this study is that it is very important to adapt the titration rate to the patient's tolerance, allowing the peritoneal cavity to adapt to the increasing pressure. As a result, the incidence of mechanical complications will be low [22].

De Vriese summarized urgent-start PD with the following points:

- The optimal time to start PD is 2 weeks after catheter insertion.

- With early break-in, there is a small increased risk of mechanical complications but no detrimental effect on mortality, peritonitis-free survival, or PD technique survival - these complications are usually relatively easy to deal with.

- PD could be an alternative to hemodialysis for urgentstart dialysis.

An important message overall is to use a titrated regimen according to the individual patient's tolerance.

\section{Presentations Reviewed}

Professor Kai-Uwe Eckardt (Risk Factors of Chronic Kidney Disease), Charité - Berlin, Germany.

kai-uwe.eckardt@charite.de.

Professor Iain Macdougall (Iron Management (Oral vs. i.v.) in Non-Dialysis Chronic Kidney Disease), King's College Hospital - London, UK. iain.macdougall@nhs.net.

Professor A.J. (Ton) Rabelink (Hypertension), Leiden University Medical Center - Leiden, The Netherlands. a.j.rabelink@lumc.nl.

Professor Patrick Murray (Acute Kidney Injury Prevention), University College Dublin - Dublin, Ireland.patrick.murray@ucd.ie.

Professor An De Vriese (Urgent Start in Peritoneal Dialysis), AZ Sint-Jan Brugge - Bruges, Belgium. an.devriese@azsintjan.be.

\section{Nephro Update Europe 2018}

Nephro Update Europe 2018 will be held on October 5 and 6, 2018, in Budapest. For more information, see www.nephro-update-europe.eu. 


\section{References}

1 Lazarus B, et al: Proton pump inhibitor use and the risk of chronic kidney disease. JAMA Intern Med 2016;176:238-246.

2 Xie Y, et al: Long-term kidney outcomes among users of proton pump inhibitors without intervening acute kidney injury. Kidney Int 2017;91:1482-1494.

3 Jung $\mathrm{CH}$, et al: The risk of chronic kidney disease in a metabolically healthy obese population. Kidney Int 2015;88:843-850

4 Rebholz CM, et al: DASH (Dietary Approaches to Stop Hypertension) diet and risk of subsequent kidney disease. Am J Kidney Dis 2016;68:853-861.

5 Kieneker LM, et al: Low potassium excretion but not high sodium excretion is associated with increased risk of developing chronic kidney disease. Kidney Int 2016;90:888-896.

6 Rogers SD, et al: Safety of intravenous ferric carboxymaltose versus oral iron in patients with nondialysis-dependent CKD: an analysis of the 1-year FIND-CKD trial. Nephrol Dial Transplant 2017;32:1530-1539.

7 Macdougall IC, et al: Renal function in patients with non-dialysis chronic kidney disease receiving intravenous ferric carboxymaltose: an analysis of the randomized FINDCKD trial. BMC Nephrol 2017;18:24.

8 Agarwal R, et al: A randomized trial of intravenous and oral iron in chronic kidney disease. Kidney Int 2015;88:905-914.
9 The SPRINT Research Group: A randomized trial of intensive versus standard blood-pressure control. N Engl J Med 2015;373:21032116.

10 Bress AP, et al: Cost-effectiveness of intensive versus standard blood-pressure control. N Engl J Med 2017;377:745-755.

11 Berlowitz DR, et al: Effect of intensive bloodpressure treatment on patient-reported outcomes. N Engl J Med 2017;377:733-744.

12 Agarwal R: Implications of blood pressure measurement technique for implementation of Systolic Blood Pressure Intervention Trial (SPRINT). J Am Heart Assoc 2017;6:e004536.

13 Townsend RR, et al: Catheter-based renal denervation in patients with uncontrolled hypertension in the absence of antihypertensive medications (SPYRAL HTN-OFF MED): a randomised, sham-controlled, proof-of-concept trial. Lancet 2017, Epub ahead of print.

14 De Leeuw PW, et al: Sustained reduction of blood pressure with baroreceptor activation therapy: results of the 6-year open follow-up. Hypertension 2017;69:836-843.

15 Meersch M, et al: Prevention of cardiac surgery-associated AKI by implementing the KDIGO guidelines in high risk patients identified by biomarkers: the PrevAKI randomized controlled trial. Intensive Care Med 2017, Epub ahead of print.
16 Zarbock A, et al: Effect of remote ischemic preconditioning on kidney injury among high-risk patients undergoing cardiac surgery: a randomized clinical trial. JAMA 2015; 313:2133-2141.

17 Zarbock A, et al: Long-term effects of remote ischemic preconditioning on kidney function in high-risk cardiac surgery patients: followup results from the RenalRIP trial. Anesthesiology 2017;126:787-798.

18 Young $\mathrm{P}$, et al; SPLIT Investigators, ANZICS CTG: Effect of a buffered crystalloid solution versus saline on acute kidney injury among patients in the intensive care unit: the SPLIT randomized clinical trial. JAMA 2015;314: 1701-1710.

19 Rowan KW, et al; PRISM Investigators: Early, goal-directed therapy for septic shock - a patient-level meta-analysis. N Engl J Med 2017;376:2223-2233.

20 Figueiredo A, et al: Clinical practice guidelines for peritoneal access. Perit Dial Int 2010; 30:424-429.

21 Ranganathan D, et al: A randomized controlled trial to determine the appropriate time to initiate peritoneal dialysis after insertion of catheter (Timely PD Study). Perit Dial Int 2017;37:420-428.

$22 \mathrm{Xu} \mathrm{D}$, et al: Urgent-start peritoneal dialysis complications: prevalence and risk factors. Am J Kidney Dis 2017;70:102-110. 